\section{Reaktionstechnische Untersuchungen zur Reaktor-Auslegung beim anaeroben Abbau komplexer Substrate am Beispiel der Methanisierung von Abläufen der Gärungsindustrie*}

\section{Alexander Aivasidis, Ulrich Giesecke, Edith Hilla und Christian Wandrey**}

Der anaerobe Abbau von komplex zusammengesetzten organischen Molekülen findet über eine recht komplizierte Nahrungskette statt, in der mindestens drei Mikroorganismen-Gruppen beteiligt sind. Dies sind im einzelnen die acidogenen, die acetogenen und die methanogenen Spezies. Neben dem breiten Spektrum und den unterschiedlichen Generationszeiten der beteiligten Mikroorganismen sowie den komplizierten Wechselwirkungen zwischen fermentativer Säurebildung und Methanogenese ist hier noch die von der Thermodynamik diktierte, regulative Rolle des Wasserstoffs bei der EssigsäureBildung durch die acetogenen Mikroorganismen zu berücksichtigen. Wird dieser nämlich nicht hinreichend schnell von den MethanBakterien verwertet, so kommt es zu einer Akkumulation von Butterund insbesondere Propionsäure im Reaktor und bei höheren Konzentrationen $\mathrm{zu}$ einer nachhaltigen Hemmung der Methan-Bildung [1].

Aufgrund des stufenweisen Abbaus mit verschiedenen BakterienPopulationen wird eine Trennung der fermentativen Säurebildung und der Methanogenese unter Verwendung mehrstufiger Reaktorkombinationen vorgeschlagen $[2,3]$. Insbesondere kommt eine zweistufige Betriebsweise zum Einsatz, bei der in der ersten Stufe (Rührkesselreaktor) die Versäuerung des fermentierbaren Materials möglichst weitgehend stattfindet. Der Ablauf gelangt dann in die zweite Stufe (z. B. Festbettreaktor oder UASB), wo die MethanBildung abläuft. Der Vorteil dieser Arbeitsweise liegt darin, daß das Potential der Säurebildung bereits in der ersten Stufe erschöpft wird

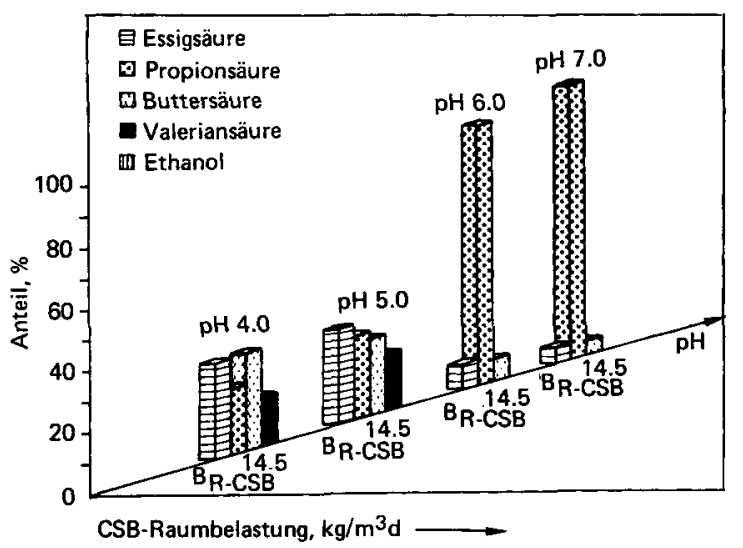

Abb. 1. Prozentuale Verteilung der Fermentationsprodukte von Brennereischlempen in Abhängigkeit vom pH-Wert in der Versäuerungsstufe.

* Vortrag von A. Aivasidis, auf dem Jahrestreffen der VerfahrensIngenieure, 17. bis 19. Sept. 1986 in Straßburg.

** Dr. A. Aivasidis, Dipl.-Chem. U. Giesecke, Prof. Dr. C. Wandrey, Kernforschungsanlage Jülich, Institut für Biotechnologie 2, Postfach 1913,5170 Jülich, und E. Hilla, KWU, Berliner Str. 295 bis 303, Postfach 962, 6050 Offenbach/M.. und die Verhältnisse in der Methan-Stufe übersichtlicher gestaltet werden.

Das zentrale Problem, auch in der anaeroben Abwasserreinigung, ist die Erzielung einer hohen Raum-Zeit-Ausbeute. Aufgrund des autokatalytischen Charakters mikrobieller Umwandlungsprozesse sollten zu diesem Zweck Biomasse-Verluste nach Möglichkeit vermieden werden. Dies geschieht konkret durch die verschiedenen Methoden zur Biomasse-Rückhaltung bzw. -Rückführung. Von der Fülle der zur Verfügung stehenden Alternativen wird von uns die Immobilisierung von Mikroorganismen durch Kolonisierung offenporiger Sintergläser der Schott-Glaswerke in Festbett-Umlaufreaktoren favorisiert $[4,5]$. Am Beispiel der ein- und zweistufigen anaeroben Behandlung von Abläufen der Gärungsindustrie, stellvertretend für komplexe Abwässer, wurden unter Einbeziehung von Festbett-Umlaufreaktoren reaktionstechnische Untersuchungen zum Einstufen- und ZweistufenProzeB mit dem Ziel durchgeführt, das optimale Konzept für die Reaktorauslegung herauszufinden.

\section{Versuchsaufbau und -durchführung}

Zwei Festbett-Umlaufreaktoren mit einem Arbeitsvolumen von 11 bzw. 181 wurden eingesetzt. Der eine wurde als einstufiges System betrieben, während der andere die Aufgabe der Methanisierung übernahm und einem Rührkesselreaktor zur Versäuerung nachgeschaltet wurde. In beiden Fällen wurde das Abwasser vor Einleiten in den Festbett-Umlaufreaktor über einen Sedimenter zur Abtrennung von suspendierten Inertteilchen und Uberschußschlamm geleitet. Sowohl für die Versuche zur Hydrolyse und Versäuerung als auch diejenigen zur Methanisierung gelangten adaptierte Mischpopulationen zum Einsatz, die aus entsprechenden Anreicherungskulturen aus Faulschlamm gewonnen wurden. Als Träger diente offenporiges Sinterglas mit einer Porosität von $50 \%$ und einer Porengrößenverteilung von 60 bis $120 \mu \mathrm{m}$ in Form von Raschig-Ringen.

Bei der untersuchten Schlempe handelte es sich um eine feststoffarme "Dünnschlempe", die durch Abdekantieren der proteinreichen Schlempe erhalten wird.

\section{Versuchsergebnisse}

Zweistufen-Prozeß: Bei konstanter Raumbelastung in der ersten Stufe wurde der Einfluß des pH-Wertes auf den Ablauf der Versäuerung und dessen Auswirkung auf die Methanisierung untersucht. In weiteren Experimenten wurde am erkannten $\mathrm{pH}-\mathrm{Optimum}$ die Raumbelastung variiert, um auch den Einfluß dieses Parameters zu erfassen. Mit zunehmender Verschiebung der Versäuerung zu niedrigeren $\mathrm{pH}$ Werten nahm der CSB-Umsatz kontinuierlich ab. Zwar wurde bei den pH-Werten 7,0 und 6,0 der Gesamt-CSB-Abbau fast einschlieBlich von der ersten Stufe getragen, die Verhältnisse kehrten sich jedoch beim Ubergang auf $\mathrm{pH} 5,0$ und 4,0 um. Unter diesen Bedingungen wurden lediglich ca. $15 \%$ des CSB-Abbaus in der ersten Stufe beobachtet, in der Hauptsache bedingt durch Abtrennung des in der Acidogenese produzierten Uberschußschlammes. Besonders interessant ist die in $\mathrm{Abb} .1$ dargestellte prozentuale Verteilung der hauptsächlich sauren Fermentationsprodukte. Daraus wird deutlich, $\mathrm{da} B$ die Produktverteilung maßgeblich vom $\mathrm{pH}$-Wert beeinflußt wird. So tritt $z$. B. Propionsäure bevorzugt im neutralen pH-Bereich $(7,0$ und 6,0 ) auf, während im sauren neben der Essigsäure auch Buttersäure gebildet wird. Tendenziell belegen diese Ergebnisse, daß die Buttersäure-Bildung durch einen neutralen $\mathrm{pH}$-Wert nachhaltiger gehemmt wird als die Propionsäure-Synthese durch einen sauren $\mathrm{pH}$ Wert. Während letztere Auswirkung ein eher gleitender Prozeß ist, wird die Buttersäure-Bildung ab einem $\mathrm{pH}$-Wert von 6,0 nahezu total gehemmt. 
Die in der Versäuerungsstufe jeweils anfallenden Abläufe der einzelnen Versuchsabschnitte wurden dem als 2. Stufe dienenden FestbettUmlaufreaktor zugeführt. Dadurch, daß der CSB-Abbau in der 1. Stufe mit abnehmendem $\mathrm{pH}$-Wert sich verschlechterte, nahm in der Methanisierung die Raumbelastung entsprechend zu. Dessen ungeachtet ließen sich in der zweiten Stufe kontinuierlich bessere Ergebnisse erzielen. Während bei neutralen $\mathrm{pH}$-Werten in der Versäuerung die CSB-Elimination in der Methanisierung maximal $20 \%$ von insgesamt $80 \%$ ausmachte, erreichte diese im weiteren Verlauf - bei saurem pH-Wert im Ablauf der 1 . Stufe - Werte von ca. $75 \%$, bei einem Gesamtabbau von mehr als $90 \%$. Offenbar sind die bei pH 4,0 bis 5,0 entstandenen sauren Abläufe besser abbaubar als diejenigen, die bei pH 6,0 bzw. 7,0 produziert wurden. Die Wasserstoff-Konzentration lag durchschnittlich bei 80 bis $100 \mathrm{ppm}$ und war gekennzeichnet durch einen besonders gleichmäßigen Kurvenverlauf. Eine kritische Prozeßüberlastung wurde nicht beobachtet.

Einstufen-Prozeß: In diesem Fall kam es in erster Linie darauf an, den Proze $B$ in einem vergleichbaren Raumbelastungsbereich zur zweistufigen Fahrweise zu betreiben. Das Fettsäure-Spektrum war während der gesamten Experimentierzeit gekennzeichnet durch zum Teil starke Schwankungen in der Konzentration der Produktsäuren, die allerdings in der Hauptsache die Essig- und Propionsäure betrafen. Ähnlichen Schwankungen war auch die Wasserstoff-Konzentration unterworfen, die im Durchschnitt bei 400 bis $600 \mathrm{ppm}$ lag und zum Teil Spitzenwerte von über $1000 \mathrm{ppm}$ erreichte. Von Interesse war die gemäß der theoretischen Vorgabe unmittelbare Korrelation von induzierten Prozeßstörungen (Verweilzeit-Schocks), vermehrtem Auftreten des Wasserstoffs sowie einer Akkumulation der Propionsäure. Die Maxima der Propionsäure- und der Wasserstoff-Konzentration in den entsprechenden Diagrammen stimmen sehr exakt miteinander überein. Der Prozeß neigte häufig zu Instabilitäten.

\section{Verfahrensvergleich und SchluBfolgerungen}

Aus den bisherigen Untersuchungen ergibt sich, $\mathrm{da} B$ eine einstufige Behandlung der Brennerei-Schlempen in einem Festbett-Umlaufreaktor im Raumbelastungsbereich von 27 bis $45 \mathrm{~kg} /\left(\mathrm{m}^{3} \mathrm{~d}\right)$ durchaus möglich ist. Wird allerdings ein kontinuierlich betriebener Rührkesselreaktor zur Durchführung einer unter definierten Bedingungen ablaufenden Versäuerung und Stufentrennung einem Festbett-Umlaufreaktor zur Methanisierung vorgeschaltet, so ergibt sich aus der Abhängigkeit der CSB-Reduktion von der Raumbelastung (Abb. 2), daß eine bessere Abbaubarkeit der Schlempe erzielt wird. Die erhaltenen CSB-Umsätze liegen beim Zweistufen-Prozeß über dem gesamten Raumbelastungsbereich durchweg höher.

Ein pH-Wert von 4,0 wurde als optimal zur Konditionierung des Schlempeabwassers in der Versäuerungsstufe für die anschließende Methanisierung erkannt. Zwar fiel der Unterschied im Abbauverhalten gegenüber $\mathrm{pH}$ 5,0 nicht sehr gravierend aus, jedoch war sowohl die Propionsäure- und Wasserstoff-Konzentration etwas geringer als auch der CSB-Umsatz leicht verbessert. Für eine optimale Auslegung des Zweistufenprozesses gemäß dem hier untersuchten Konzept sollte bei Verzicht auf eine Biomasse-Rückführung eine Verweilzeit von

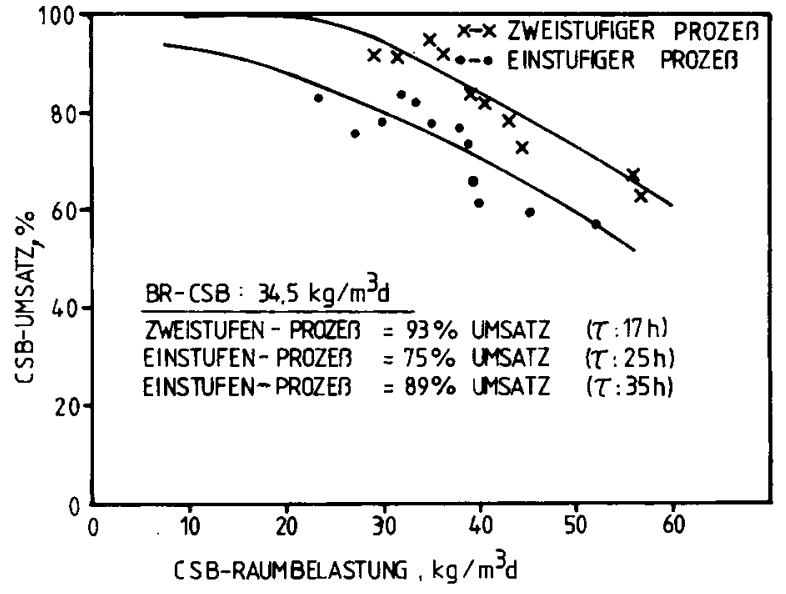

Abb. 2. Einfluß der CSB-Raumbelastung auf den CSB-Umsatz des Ein- und Zweistufen-Prozesses.

$2 \mathrm{~d}$ in der Versäuerung zur weitgehenden Erschöpfung des Säurebildungspotentials und von $0,75 \mathrm{~d}(17 \mathrm{~h})$ in der Methanisierung eingehalten werden. Die Einbindung eines Sedimenters im Ablauf der Versäuerung zur Abtrennung von Inertteilchen und Überschußschlamm aus der 1. Stufe und Vermeidung einer äußerst unerwünschten Akkumulation in der zweiten Stufe hat sich als besonders zweckmäßig erwiesen. Diese Maßnahme trägt entscheidend zur Verbesserung der Betriebslangzeitstabilität bei. In der Praxis konnte der zur Methanisierung dienende Festbett-Umlaufreaktor, bei vergleichbarer Raumbelastung zum einstufigen Prozeß, über eine um den Faktor vier längere Zeit gegenüber dem letzteren stabil betrieben werden.

Eingegangen am 30. Oktober 1986

[1] Lettings, G.; van Velsen, L.; de Zeeuw, W.; Hobma, S. W., in: Anaerobic Digestion, S. 167/168, Applied Science Publishers Ltd., London 1980.

[2] Kunst, S.; Mudrack, K.: Gas Wasserfach, Wasser Abwasser 124 (1983) Nr. 2, S. 77/86.

[3] Sutton, P. M.; Li, A.: Water Sci. Technol. 15 (1983) S. 333/ 334.

[4] Aivasidis, A.; Wandrey, C.: Pat. Anmeldung Kernforschungsanlage Jülich, P 3247117.3 v. 21. 12. 1982.

[5] Aivasidis, A.; Wandrey, C.: Ein Glasschwamm als Bakterienspeicher - Abwasserreinigung ohne Sauerstoff. Bericht der KFA Jülich, Nr. 1900.

Schlüsselworte: Reaktor-Auslegung, aerober Abbau, Methanisierung, Gärungsindustrie.

Das vollständige Manuskript dieser Arbeit umfaßt 25 Seiten mit 17 Abbildungen und 3 Tabellen. Es ist als Fotokopie oder Mikrofiche MS 1580/87 erhältlich. Bestellkarten finden Sie am Schluß dieses Heftes. 